$25 \rightarrow$

Das carbenanaloge Verhalten des Phosphors in 5 sollte auf dessen erhöhte Nucleophilie, hervorgerufen durch die tert-Butylgruppe, zurückzuführen sein.

Eingegangen am 29. Januar 1982 [Z 121] Das vollständige Manuskript dieser Zuschrift erscheint in: Angew. Chem. Suppl. 1982, 1009-1015

[2] M. Baudler, Pure Appl. Chem. 52 (1980) 755.

[7] M. Hesse, U. Klingebiel, noch unveroffentlicht.

[8] E. Fluck, Top. Phosphorus Chem. 10 (1980) 193; R. Appel, F. Knoll, J. Ruppert, Angew. Chem. 93 (1981) 771; Angew. Chem. Int. Ed. Engl. 20 (1981) 731 .

[9] E. Niecke, R. Rüger, Nachr. Chem. Tech. Lab. 29 (1981) 754.

[10] 1, Ausb. $30 \%, \mathrm{Kp}=116^{\circ} \mathrm{C} / 0.01$ Tor, $\mathrm{MS}(70 \mathrm{eV}): \mathrm{m} / \mathrm{z} 358\left(35 \%, \mathrm{M}^{+}\right)$; ${ }^{31} \mathrm{P}-\mathrm{NMR}$ (in $\mathrm{C}_{6} \mathrm{H}_{6}, \mathrm{H}_{3} \mathrm{PO}_{4}$ int.): $\delta=-93.3\left(\mathrm{P}_{\mathrm{A}}\right),-101.5\left(\mathrm{P}_{\mathrm{B}}\right), J_{\mathrm{PP}}=206$ $\mathrm{Hz}$.

[11] 6, Ausb. $\approx 15 \%, \mathrm{Fp}=147^{\circ} \mathrm{C}, \mathrm{MS}(70 \mathrm{eV}): \mathrm{m} / 2364\left(60 \%, M^{+}\right) ;{ }^{1} \mathrm{H}-\mathrm{NMR}$ (in $\mathrm{C}_{6} \mathrm{H}_{6}, \mathrm{TMS}$ int.): $\delta=0.39$ ( $\left.\mathrm{SiMe}_{2}\right), 1.37(t \mathrm{BuN}), 2.28$ (CMe).

\section{Komplexe von Kronenethern mit Uroniumsalzen: \\ Herstellung und Kristallstruktur von \\ [18]Krone-6-Uroniumnitrat (1/1) und \\ Benzo[27]krone-9-Uroniumperchlorat (1/1)}

Von Jos W. H. M. Uiterwijk, Sybolt Harkema,

David N. Reinhoudt, Kari Daasvatn,

Herman J. den Hertog Jr. und Jan Geevers

Als Teil von Untersuchungen zur Synthese von Rezeptormolekülen für Harnstoff haben wir kürzlich den ersten kristallinen Komplex von Harnstoff mit einem makrocyclischen Polyether, [18]Krone-6 1, hergestellt ${ }^{[1,2]}$.

Um zu prüfen, ob die Stabilität von Harnstoffkomplexen durch Protonierung (am Sauerstoffatom ${ }^{[5]}$ ) erhöht wird, synthetisierten wir Komplexe von 1 mit Uroniumnitrat $(1: 1)$, -pikrat $(1: 1)$ und -p-toluolsulfonat $(1: 2)$ sowie einen Komplex von Benzo[27]krone-9 2 mit Uroniumperchlorat $(1: 1)$. Die beiden Titelverbindungen wurden bei 168 bzw. $269 \mathrm{~K}$ durch Röntgen-Strukturanalyse untersucht.

In beiden Fällen wird der Ort der Protonierung durch die Komplexierung nicht beeinflußt. Interessant an 1. Uroniumnitrat $(1 / 1)$ ist die recht ungewöhnliche Konformation des Polyetherringes $\left(a g^{+} a a g^{-} a a^{+} g^{+} a g^{+} a\right.$ $\left.a g^{-} a a^{+} g^{+}\right)$; in $1 \cdot$ Harnstoff $(1 / 5)$ und $1 \cdot S$-tert-Butylthiouroniumperchlorat $(1 / 2)$ sind die Ringe dagegen zentrosymmetrisch mit $\left(a g^{+} a a^{+} a g^{+} g^{+} a\right)$ - bzw. $\left(a g^{+} a\right.$ $\left.a g^{-} a a^{+} a\right)$-Konformation ${ }^{[1]}$.

1. Uroniumnitrat $(1 / 1)$ enthält drei $\mathrm{N}-\mathrm{H}$... O-Wasserstoffbrücken zu den O-Atomen 1, 2 und 4 des Polyetherrings. Das Gastmolekül befindet sich außerhalb der Ebene des Kronenethers (Fig. 1).

In 2. Uroniumperchlorat $(1 / 1)$ ist das Gastmolekül dagegen völlig vom Kronenether umhüllt; es befindet sich nahezu in der besten Ebene durch die Ether-Sauerstoff-

["] Drs. J. W. H. M. Uiterwijk [a], Dr. S. Harkema [a], Prof. Dr. Ir. D. N. Reinhoudt [b], Dr. K. Dassvatn [b], Dr. H. J. den Hertog Jr. [b], J. Geevers [b] Laboratories of Chemical Physics" and Organic Chemistry Twente University of Technology

P.O. Box 217, NL-7500 AE Enschede (Niederlande)

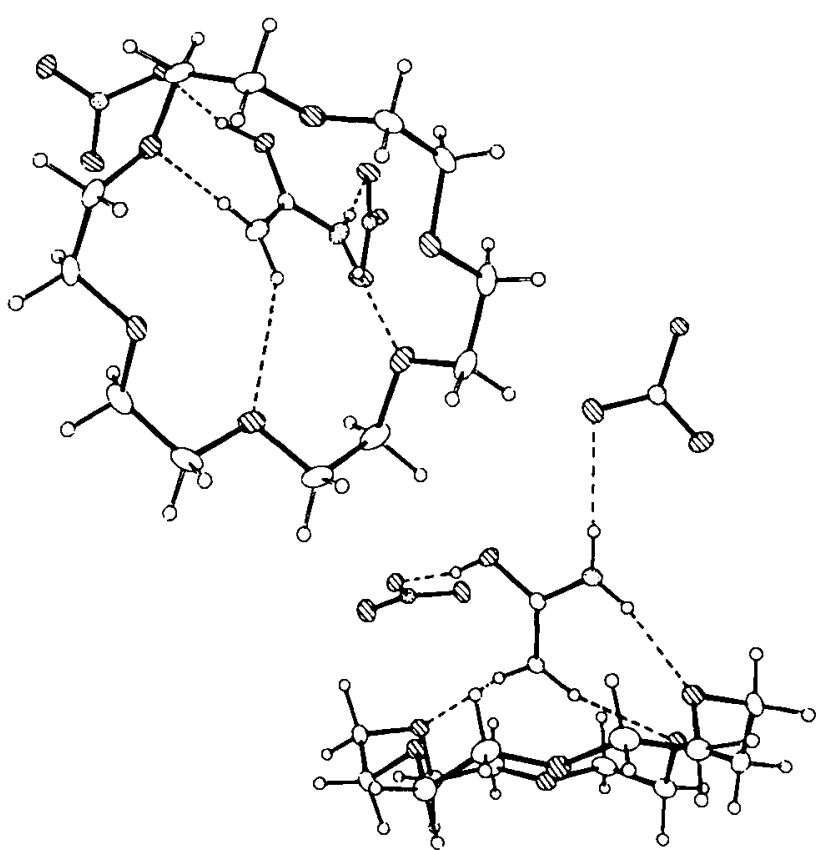

Fig. 1. 1:1-Komplex von [18]Krone-6 1 und Uroniumnitrat im Kristall, von "oben" und "von der Seite" gesehen. $P 2_{1} / n, a=20.304(9), b=11.604(6)$, $c=8.181(3) \dot{A}, \beta=93.95(4)^{\circ}$.

atome. Der Polyetherring hat die Konformation asa $a g^{-} g^{-} a g^{+} a a g^{-} a a g^{-} g^{-} a g^{+} a a g^{-} a a g^{-} g^{-} g^{-} g^{-} a(s$ bedeutet synperiplanar, d. h. einen Torsionswinkel von $0^{\circ}$, der durch den anellierten Benzolring verursacht wird).

Alle fünf Uronium-H-Atome bilden H-Brücken zu RingO-Atomen (Fig. 2). Die Konformation des Polyetherringes unterscheidet sich zwar betrăchtlich von derjenigem im isoelektronischen 2 . Guanidiniumperchlorat $(1 / 1)^{[16]}$ (asa $\left.a g^{+} a g^{-} g^{-} g^{-} a g^{-} a a g^{-} g^{-} a g^{+} a g^{+} g^{+} a a g^{-} a a g^{-} a\right)$, doch nehmen die O-Atome des Ringes, die sich an den $\mathrm{H}$ Brücken beteiligen, ungefähr die gleichen Positionen relativ zum zentralen Kation ein.

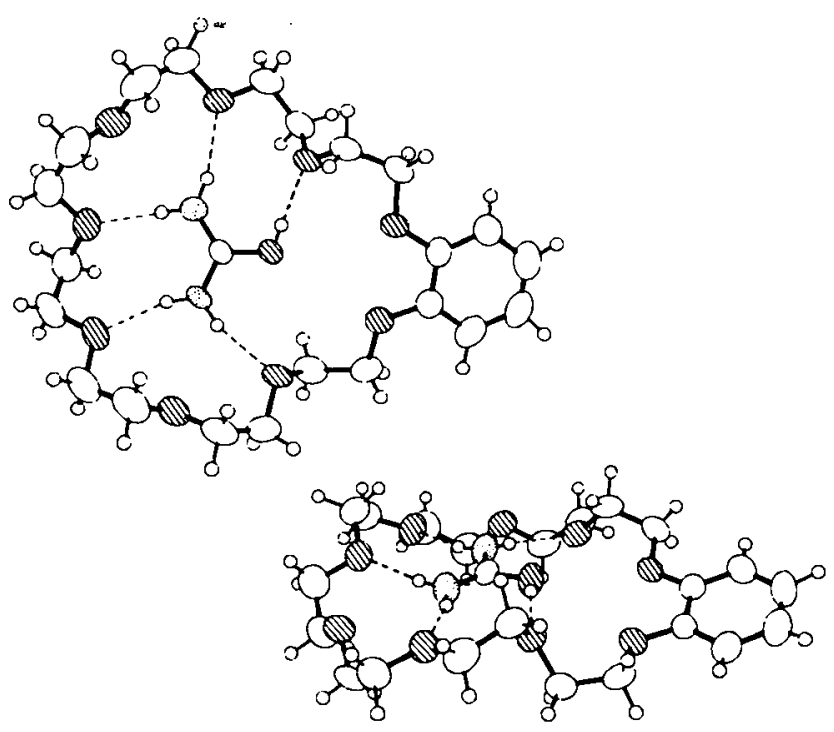

Fig. 2. 1:1-Komplex von Benzo[27]krone-9 2 und Uroniumperchlorat im Kristall, von "oben" und "von der Seite" gesehen. Pí, $a=13.866(5)$, $b=12.585(7), c=9.926(6) \dot{A}, \alpha=99.59(3), \beta=74.91(6), \gamma=116.52(3)^{\circ}$

Unsere Befunde zeigen, daß Kronenether in Komplexen mit polyfunktionellen organischen Kationen je nach Typ 
des Kations und des Anions verschiedene Konformationen einnehmen können.

Eingegangen am 24. Februar 1982 [Z 119] Das vollstăndige Manuskript dieser Zuschrift erscheint in: Angew. Chem. Suppl. 1982, 1100-1107

[1] S. Harkema, G. J. van Hummel, K. Daasvatn, D. N. Reinhoudt, J. Chem. Soc. Chem. Commun. 1981, 368.

[2] Komplexe von Harnstoff mit linearen Polyethern: W. Rasshofer, F Vögtle, Tetrahedron Lett. 1978, 309; G. Oepen, F. Vögtle, Liebigs Ann. Chem. 1980, 512; U. Heimann, F. Vogtle, Chem. Ber. 112 (1979) 3034; F. Vögtle, G. Oepen, W. Rasshofer, Liebigs Ann. Chem. 1979, 1577

[5] Neutronenstreuung: J. E. Worsham, W. R. Busing, Acta Crystallogr. B 25 (1969) 572; Röntgenbeugung: S. Harkema, D. Feil, ibid. B 25 (1969) 589.

[11] J. W. H. M. Uiterwijk, S. Harkema, G. J. van Hummel, J. Geevers, D. N. Reinhoudt, Acta Crystallogr. B, im Druck.

[16] J. W. H. M. Uiterwijk, S. Harkema, J. Geevers, D. N. Reinhoudt, J. Chem. Soc. Chem. Commun. 1982, 200.

Synthese neuartiger Iridium- und Rhodiumkomplexe mit Diphenylphosphinito- und DimethylphosphitoLiganden **

Von J. Andrew S. Duncan, David Hedden,

D. Max Roundhill, T. Anthony Stephenson* und Malcolm D. Walkinshaw

Man kennt bereits einige Übergangsmetallverbindungen mit Liganden $\mathrm{R}_{2} \mathrm{POHOPR}_{2}(\mathrm{R}=\mathrm{Ph}, \mathrm{OMe}, \mathrm{OEt})$, die $\mathrm{H}-$ Brücken enthalten ${ }^{[1]}$; meist wurden Palladium(II)- und Platin(II)-Komplexe untersucht. Wir berichten nun über verwandte Iridium- und Rhodium-Verbindungen ${ }^{[2]}$

Die Umsetzung von $\left[\{\operatorname{IrCl}(\operatorname{cod})\}_{2}\right](\operatorname{cod}=1,5$-Cyclooctadien) mit $\mathrm{Ph}_{2} \mathrm{PCl}$ im Überschuß führt in wäßrigem Methanol $(1: 7, \mathrm{v} / \mathrm{v})$ bei Raumtemperatur (d. h. Umsetzung mit $\mathrm{Ph}_{2} \mathrm{P}(\mathrm{O}) \mathrm{H}+\mathrm{HCl}$ ) zum neutralen, monomeren Iridium(III)-Komplex $1^{[3]}$. In Lösung spaltet 1 schnell das Dien ab und bildet in Gegenwart zweizăhniger Liganden $\mathrm{L}_{2}$ mit hohen Ausbeuten Verbindungen 2a-c, die nicht zu weiterer Abgabe von Liganden neigen. 2b setzt sich mit $\mathrm{HBF}_{4}$ zum kationischen Komplex 3b um, der sich beim Wiederauflösen in $\mathrm{CHCl}_{3}$ unter HF-Abspaltung schnell zu $4 \mathrm{~b}$ umlagert. $4 \mathrm{~b}$ kann auch aus $2 \mathrm{~b}$ und $\mathrm{BF}_{3} \cdot \mathrm{Et}_{2} \mathrm{O}$ erhalten werden. Vorläufige Untersuchungen zeigen, daß 1 mit der Pla tin-Spezies 5 den ungewöhnlichen Komplex $6^{[4]}$ mit zwei verschiedenen Metallen ergibt. Auf diesem Weg sollten weitere derartige Verbindungen zugänglich sein

Ähnlich setzte sich der Iridium-Komplex 7 mit $(\mathrm{MeO})_{2} \mathrm{P}(\mathrm{O}) \mathrm{H}$ fast quantitativ zum kationischen Komplex 8 um, dessen Konfiguration ' $\mathrm{H}$-NMR-spektroskopisch ermittelt wurde.

Verwandte (dien)-Rhodium(1)-Verbindungen bilden dagegen mit $\mathbf{R}_{\mathbf{2}} \mathrm{P}(\mathrm{O}) \mathrm{H}$-Liganden unter leichtem Dien-Austausch andersartige Produkte. Darüber hinaus hăngt die Art des Produkts z. B. der Reaktion von $\left[\{R h C l(\operatorname{cod})\}_{2}\right]$ mit $\mathrm{Ph}_{2} \mathrm{PCl}$ im Überschuß in wäßrigem Methanol kritisch vom Verhältnis der Solventien ab. Bei niedrigem Wasser : Methanol-Gehalt $(1: 15, \mathrm{v} / \mathrm{v})$ entstanden nur die wohlbekannten Kationen $\left[\mathrm{Rh}\left(\mathrm{Ph}_{2} \mathrm{POMe}\right)_{4}\right]^{+}$und $\left[\mathrm{RhH}(\mathrm{Cl})\left(\mathrm{Ph}_{2} \mathrm{POMe}\right)_{4}\right]^{+}$

[*] Dr. T. A. Stephenson, Dr. M. D. Walkinshaw, J. A. S. Duncan Department of Chemistry, University of Edinburgh Edinburgh EH9 3JJ (Schottland)

Prof. D. M. Roundhill, D. Hedden

Department of Chemistry, Washington State University Pullman, Washington (USA)

[**] Diese Arbeit wurde vom Science and Engineering Research Council (Stipendium für J. A. S. D.), dem Petroleum Research Fund (Grant No. $11633 \mathrm{AC} 3$ ) und der NATO (Grant No. 1725) unterstützt. Wir danken Johnson-Matthey Ltd. (T. A. S.) und Engelhard (D. M. R.) für Leihgaben von $\mathrm{RhCl}_{3}$ und $\mathrm{IrCl}_{3}$

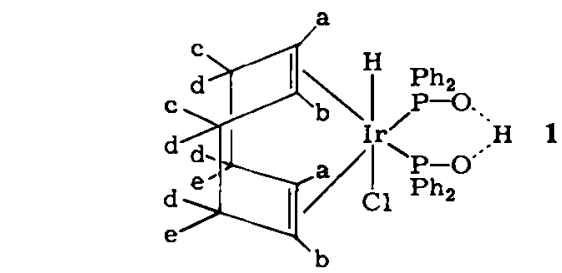

$5 \mathrm{NEt}_{2} \mathrm{H}_{2}\left[\mathrm{Pt}\left(\mathrm{S}_{2} \mathrm{CNEt}_{2}\right)\left(\mathrm{Ph}_{2} \mathrm{PS}\right)_{2}\right] / \mathrm{L}_{2}$

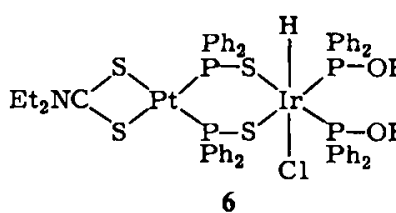

6<smiles>C[18CH][18CH]</smiles>

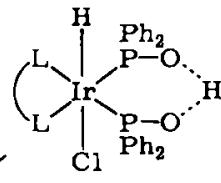
(2b) $\mathrm{BF}_{3} \cdot \mathrm{Et}_{2} \mathrm{O}$

3b

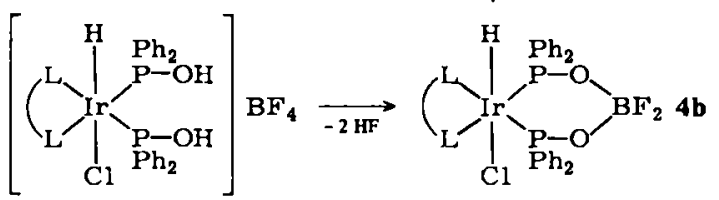

a, $L_{2}=\mathrm{Ph}_{2} \mathrm{P}\left(\mathrm{CH}_{2}\right)_{2} \mathrm{PPh}_{2} ;$ b, $\mathrm{L}_{2}=\mathrm{Ph}_{2} \mathrm{As}\left(\mathrm{CH}_{2}\right)_{2} \mathrm{AsSh}_{2}$; c, $\mathrm{L}_{2}=\mathrm{PhS}\left(\mathrm{CH}_{2}\right)_{2} \mathrm{SPh}$

$$
\begin{gathered}
{\left[\mathrm{Ir}(\mathrm{MeCN}) \mathrm{CO}\left(\mathrm{PPh}_{3}\right)_{2}\right] \mathrm{ClO}_{4}} \\
7 \stackrel{+}{(\mathrm{MeO})_{2} \mathrm{PH}_{\mathrm{O}}}
\end{gathered}
$$

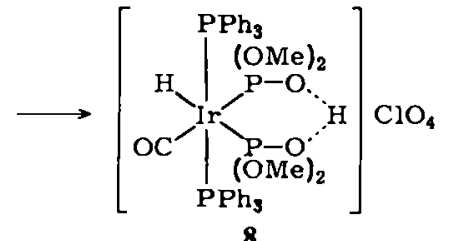

die als $\mathbf{P F}_{6}$-Salze isoliert wurden ${ }^{[5]}$. Bei höherem Wasser : Methanol-Gehalt $(1: 7, \mathrm{v} / \mathrm{v})$, kurzer Reaktionszeit bei Raumtemperatur und anschließender Zugabe von Salzen mit großen Kationen bilden sich die neuartigen zweikernigen anionischen Komplexe $\mathrm{M}\left[\mathrm{Rh}_{2} \mathrm{Cl}_{5}\left(\left(\mathrm{Ph}_{2} \mathrm{PO}_{2} \mathrm{H}\right)_{2}\right]\right.$ $\left(\mathrm{M}=\mathrm{AsPh}_{4}, \mathrm{Ph}_{3}\left(\mathrm{PhCH}_{2}\right) \mathrm{P}, \mathrm{Cs}\right) \mathrm{mit}$ drei Chloro-Brücken in hohen Ausbeuten. Im AsPh $\mathrm{Ph}_{4}$-Salz (Fig. 1) sind die $\mathrm{O} \cdots \mathrm{O}$ Abstände von $2.4 \dot{A}$ in Einklang mit denen in anderen Komplexen mit symmetrischen $\mathrm{H}$-Brücken ${ }^{[1]}$.

Bei Umsetzungen von Salzen des Typs [Rh(dien) $\left.\mathrm{L}_{2}\right] \mathrm{ClO}_{4}$ mit $\mathrm{H}_{2}$ und danach mit $(\mathrm{MeO})_{2} \mathrm{P}(\mathrm{O}) \mathrm{H}$ (Molverhältnis $1: 2$ ) ließen sich mehrere luftbeständige kationische Rhodium(I)-Komplexe $\left[\mathrm{RhL}_{2}\left(\mathrm{P}(\mathrm{OMe})_{2} \mathrm{OH}\right)_{2}\right] \mathrm{ClO}_{4}\left(\mathrm{~L}_{2}=2 \mathrm{PPh}_{3}, 2\right.$ $\left.\mathrm{PMePh}_{2}, \mathrm{Ph}_{2} \mathrm{P}\left(\mathrm{CH}_{2}\right)_{2} \mathrm{PPh}_{2}\right)$ isolieren, in denen die trikoor

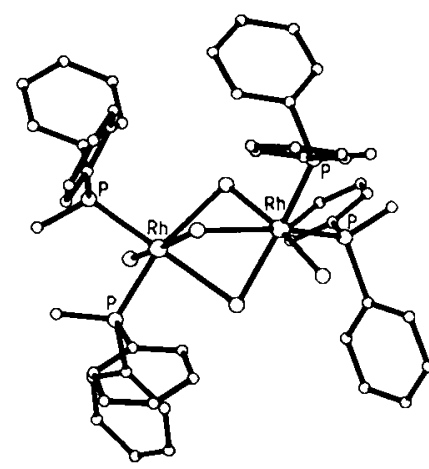

Fig. 1. Struktur des Anions von $\mathrm{AsPh}_{4}\left[\mathrm{Rh}_{2} \mathrm{Cl}_{5}\left(\left\langle\left.\mathrm{Ph}_{2} \mathrm{PO}\right|_{2} \mathrm{H}\right)_{2}\right]\right.$ im Kristall. Pi $a=12.157(4), \quad b=16.191(5), \quad c=20.523(8) \quad \dot{\mathrm{A}}, \alpha=96.02(3), \quad \beta=99.88(2)$ $\gamma=96.10(2)^{\circ}, U=3926 \dot{A}^{3}, Z=2$. Verfeinerung bis $R=0.084,4400$ unabhän gige Reflexe $\left(\mathrm{Mo}_{\mathrm{Ka}}\right)$ mit $I>2.5 \sigma(I)$, Rh-Atome mit anisotropen Temperaturfaktoren, idealisierte Phenylringe; fehlgeordnete Solvensmoleküle $\left(\mathrm{CH}_{2} \mathrm{Cl}_{2}\right)$ noch nicht vollstandig verfeinert. 